\title{
Review of: "Halpern-type relaxed inertial algorithms with Bregman divergence for solving variational inequalities"
}

\author{
Xingxing $\mathrm{Ju}^{1}$ \\ 1 Southwest University in Chongqing
}

Potential competing interests: The author(s) declared that no potential competing interests exist.

Manuscript Title: Halpern-type relaxed inertial algorithms with Bregman

divergence for solving variational inequalities

Corresponding Author: Pongsakorn Sunthrayuth

Recommendation: The paper is probably publishable, but should be reviewed again in revised form before it is accepted.

Additional Comments: In the present work, the authors introduce and analyze two new inertial-like algorithms with Bregman divergence for solving pseudomonotone variational inequalities in a real Hilbert space. The first algorithm is inspired by Halpern-type iteration and subgradient extragradient method and the second algorithm is inspired by Halpern-type iteration and Tseng's extragradient method. This article has considerable values, however, it is not outstanding. I would recommend some changes to be taken into account in the manuscript before acceptance for publication. There are several points to improve.

1. In page 13, The definition of the same definition is too redundant.

2. Please supplement the latest literature on this subject.

3. If you can provide a practical example, it will be very persuasive.

4. There are many grammatical errors throughout the paper which should be corrected. The use of the words 'the' and 'a' were consistently left out or misused.

5. It is suggested that the derivation of many other formulas should be detailed. 